\title{
Electrical Coupling Between Glial Cells in the Rat Retina
}

\author{
PAUL W. CEELEN, ${ }^{1}$ AMBER LOCKRIDGE, ${ }^{2}$ AND ERIC A. NEWMAN, ${ }^{1 *}$ \\ ${ }^{1}$ Department of Neuroscience, University of Minnesota, Minneapolis, Minnesota \\ ${ }^{2}$ St. Paul Academy and Summit School, St Paul, Minnesota
}

KEY WORDS astrocyte; Müller cell; space constant; barium; octanol; simulation

\begin{abstract}
The strength of electrical coupling between retinal glial cells was quantified with simultaneous whole-cell current-clamp recordings from astrocyte-astrocyte, astrocyte-Müller cell, and Müller cell-Müller cell pairs in the acutely isolated rat retina. Experimental results were fit and space constants determined using a resistive model of the glial cell network that assumed a homogeneous two-dimensional glial syncytium. The effective space constant (the distance from the point of stimulation to where the voltage falls to $1 / e$ ) equaled $12.9,6.2$, and $3.7 \mu \mathrm{m}$, respectively for astrocyte-astrocyte, astrocyte-Müller cell, and Müller cell-Müller cell coupling. The addition of $1 \mathrm{mM} \mathrm{Ba}{ }^{2+}$ had little effect on network space constants, while $0.5 \mathrm{mM}$ octanol shortened the space constants to 4.7, 4.4, and $2.6 \mu \mathrm{m}$ for the three types of coupling. For a given distance separating cell pairs, the strength of coupling showed considerable variability. This variability in coupling strength was reproduced accurately by a second resistive model of the glial cell network (incorporating discrete astrocytes spaced at varying distances from each other), demonstrating that the variability was an intrinsic property of the glial cell network. Coupling between glial cells in the retina may permit the intercellular spread of ions and small molecules, including messengers mediating $\mathrm{Ca}^{2+}$ wave propagation, but it is too weak to carry significant $\mathrm{K}^{+}$spatial buffer currents. GLIA 35:1-13, 2001. ๑ 2001 Wiley-Liss, Inc.
\end{abstract}

\section{INTRODUCTION}

Glial cells in the central nervous system (CNS) are coupled extensively by gap junctions (Ransom, 1995). This coupling is believed to underlie several important glial cell processes, including the regulation of extracellular $\mathrm{K}^{+}$by spatial buffering (Orkand et al., 1966; Newman, 1995), the propagation of intercellular $\mathrm{Ca}^{2+}$ waves (Finkbeiner, 1993; Enkvist and McCarthy, 1992; Newman, 2001a), and the regulation of intracellular ion levels (Loewenstein, 1981; Rose and Ransom, 1997). Despite its importance, few quantitative studies of coupling between glial cells in the CNS have been conducted. Electrical coupling between glia has been characterized in cell culture (Kettenmann and Ransom, 1988; Ransom and Kettenmann, 1990; Von Blankenfeld et al., 1993), but, with just one exception (Mobbs et al., 1988), the strength of coupling in intact CNS tissue has not been quantified in detail.

Two types of macroglial cells are present in the mammalian retina: astrocytes and Müller cells (Newman, 2001b) (Fig. 1). Astrocytes are confined to the nerve fiber layer at the vitreal surface of the retina. Müller cells, radial glial cells, span the entire depth of the retina. Coupling between glial cells in the mammalian retina has been characterized in tracer studies (Robinson et al., 1993; Zahs and Newman, 1997). Astrocytes are coupled to other astrocytes by gap junctions that pass both Neurobiotin and Lucifer yellow. Astrocytes are coupled to Müller cells, in contrast, by junctions that permit diffusion of the small tracer Neurobiotin from astrocytes to Müller cells, but not from Müller cells to astrocytes. In addition, the larger, negatively charged tracer Lucifer yellow does not pass through these junctions in either direction.

Grant sponsor: National Institutes of Health; Grant number: EY04077.

*Correspondence to: Eric A. Newman, Department of Neuroscience, University of Minnesota, 6-145 Jackson Hall, 321 Church Street SE, Minneapolis, MN 55455. E-mail: ean@umn.edu

Received 29 December 2000; Accepted 11 April 2001 


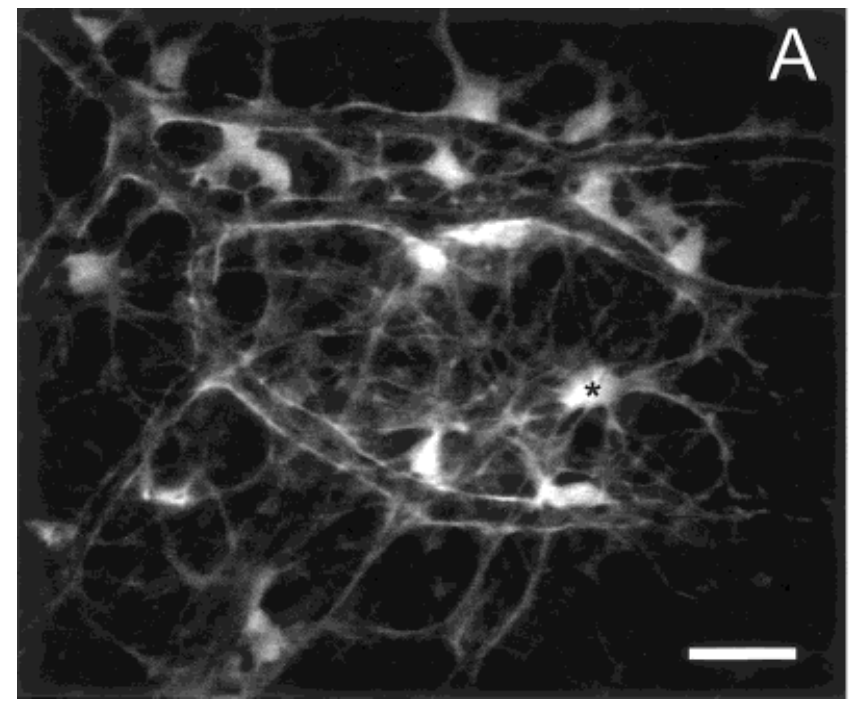

Fig. 1. Glial cells of the rat retina, visualized by injection of the tracer Neurobiotin. The tracer, introduced into a single astrocyte through a patch pipette, diffused into neighboring astrocytes and Müller cells through gap junctions. A: Confocal image at the vitreal surface of the retina, showing Neurobiotin-filled astrocytes. Asterisk indicates the injected cell. B: Image within the inner plexiform layer

The strength of glial cell coupling in the mammalian retina remains unknown. Are astrocytes coupled to each other strongly enough to mediate spatial buffer currents? Are junctions between astrocytes and Müller cells electrically rectifying? We have addressed these questions in an electrophysiological study of glial cell coupling in the acutely isolated rat retina. Simultaneous whole-cell current-clamp recordings were made from pairs of glial cells at the vitreal surface of the retina. Experimental results have been fit using two resistive models of glial cells in the retina, and estimates of the space constants of the glial networks have been made.

\section{MATERIALS AND METHODS Preparation}

Recordings were made from isolated whole-mount retinas of the rat. The preparation has been described previously (Zahs and Newman, 1997). Briefly, male Long-Evan rats $(250-400 \mathrm{~g})$ were deeply anesthetized with sodium pentobarbital administered intraperitoneally, and the eyes were removed. Retinas were removed from the back of the eye, cut in half, and stored in oxygenated Ringer's solution for later use. A piece of retina was incubated for $16 \mathrm{~min}$ at room temperature in collagenase/dispase $(2 \mathrm{mg} / \mathrm{ml})$ and DNase $(0.1 \mathrm{mg} /$ $\mathrm{ml}$ ) in Ringer's solution to digest the basal lamina at the inner surface of the retina and to facilitate removal of the vitreous humor, which was peeled off the retina with forceps after $8 \mathrm{~min}$ in the incubation solution. The incubation solution also contained $10 \mu \mathrm{M}$ bisBenzimide Hoechst No. 33342, a membrane-permeant nuclear

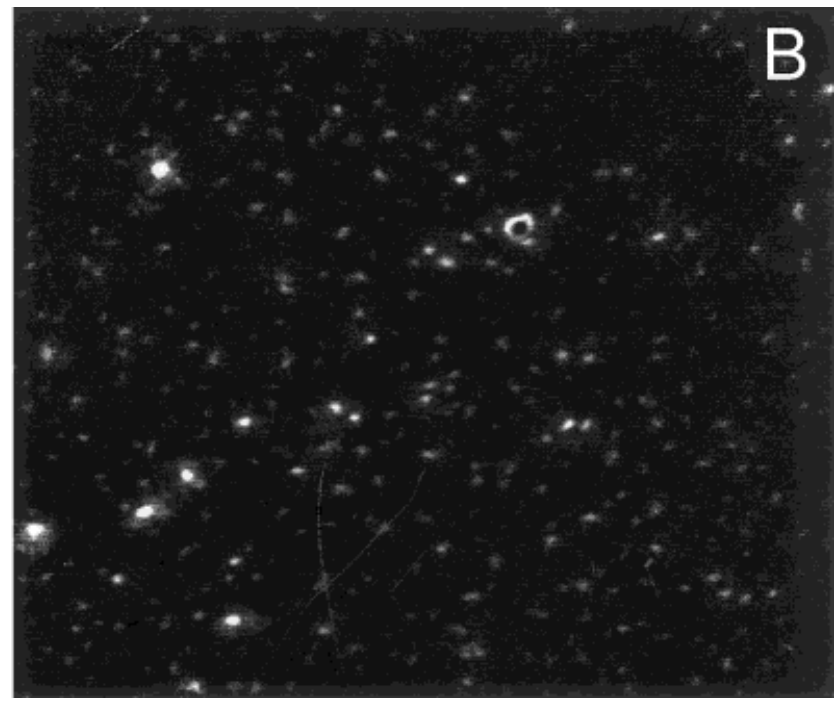

at the same retinal location as A, showing Neurobiotin-filled radial processes of Müller cells. Each large or small white spot represents a Müller cell. Note that the density of Müller cells is much greater than the density of astrocytes. Methods describing this type of tracer experiment are given in Zahs and Newman (1997). Images courtesy of Dr. Kathleen R. Zahs. Scale bar $=25 \mu \mathrm{m}$.

stain. After incubation, the retina was attached to a polycarbonate membrane filter (vitreal side up) by suction. The membrane filter had a $\sim 2 \times 2$-mm window cut into it so that the retina could be viewed by transillumination. The retina and filter were held in place in a superfusion chamber by a ring of platinum wire. The retina was superfused at $\sim 2 \mathrm{ml} / \mathrm{min}$ with HEPESbuffered Ringer's solution at $\sim 23^{\circ} \mathrm{C}$.

\section{Recording Procedures}

Simultaneous whole-cell recordings were made from pairs of retinal glial cells at the vitreal surface of the retina with patch pipettes having resistances of 6-12 $\mathrm{M} \Omega$ (before patching onto cells). Oblique transillumination was used to visualize the retinal surface, which had a pebbly, textured appearance when viewed through a long working distance $20 \times$ objective.

Recordings from astrocytes were obtained by first locating a bisBenzimide-labeled astrocyte nucleus with ultraviolet (UV) epifluorescence and then patching onto the retinal surface directly above the nucleus. Müller cell recordings were obtained from cell endfeet by patching onto the retinal surface at random locations.

Recorded cells were identified by filling them with Lucifer yellow, contained in the patch pipette. Astrocytes were identified by their flattened polymorphic somata and multiple radial processes that lay almost entirely within the nerve fiber layer. Müller cells were identified by their expanded endfoot at the retinal surface, their principal radial process projecting down- 
ward through the inner plexiform layer, and their soma in the inner nuclear layer.

Electrical coupling between pairs of cells was characterized only if both cells of the pair were positively identified by Lucifer yellow fills and both recordings were stable for the duration of the experiment, typically lasting 10-25 min. Astrocyte and Müller cell recordings were considered stable if their resting membrane potentials remained more negative than -75 and $-80 \mathrm{mV}$, respectively. Coupling between pairs of cells was determined by injecting a positive 0.5-nA current into one cell of the pair and recording the resulting voltage change in the second cell.

\section{Solutions}

The HEPES-buffered Ringer's superfusate solution contained (in $\mathrm{mM}$ ): $\mathrm{NaCl}, 140 ; \mathrm{KCl}, 2.5 ; \mathrm{CaCl}_{2}, 3.0$; $\mathrm{MgCl}_{2}, 0.5$; D-glucose, 15.0; and HEPES, 5. It was adjusted to $\mathrm{pH} 7.4$ with $\mathrm{NaOH}$ and equilibrated with $100 \% \mathrm{O}_{2} ; 1 \mathrm{mM} \mathrm{BaCl}_{2}$ and $0.5 \mathrm{mM}$ octanol were added directly to the Ringer's solution. The patch pipette solution contained (in $\mathrm{mM}$ ): $\mathrm{KCl}, 124.2 ; \mathrm{MgCl}_{2}, 1.0$; $\mathrm{CaCl}_{2}, 2.0$; HEPES, 10.0; EGTA, 5.0; and Lucifer yellow $\mathrm{CH}, 1 \mathrm{mg} / \mathrm{ml}$. It was adjusted to $\mathrm{pH} 7.1$ with $\mathrm{KOH}$.

Results are given as mean \pm SEM (number of samples). Statistical significance was assessed using Student's $t$-test for unpaired samples.

\section{Resistive Models of Glial Cell Coupling}

We developed two different two-dimensional resistive models of glial cell coupling with which to analyze the experimental results. Analytical solutions have been used in previous studies of coupling in two-dimensional networks of photoreceptors (Lamb and Simon, 1976; Detwiler and Hodgkin, 1979) and amphibian Müller cells (Mobbs et al., 1988). Because of the geometric complexity of coupling between glial cells in the mammalian retina, however, we chose to model the system using discrete, two-dimensional networks of ohmic resistors. The software program Pspice (OrCAD Release 9, Portland, OR), run on a PC-compatible computer, was used to calculate the current flow through the resistive networks. Our two models are described below and are illustrated in Figure 2. Model parameters are summarized in Table 1.

\section{Uniform resistive network model}

The glial cell network is modeled as a homogeneous two-dimensional syncytium, rather than a network of distinct cells. The syncytium is modeled by a uniform array of resistors (Fig. 2A, blue rectangles, AA) that represent both the internal resistance of astrocytes and the resistance of the gap junctions between the astrocytes. The distance between adjacent nodes in the ar- ray represents $10 \mu \mathrm{m}$ on the retinal surface and a $5 \times$ 5 array of nodes represents a single astrocyte. This corresponds to an astrocyte density of 200 cells $/ \mathrm{mm}^{2}$, the average density measured in the rat retina (ChanLing and Stone, 1991). Each node (Fig. 2A, A1, A2 . . ) represents an intracellular compartment in the astrocyte syncytium. The entire resistive model consists of a $24 \times 26$ array of nodes, an array representing approximately 25 astrocytes.

Each node in the array is connected to ground by a shunt resistor (Fig. 2A, green rectangles, $\mathrm{AMG}$ ) that represents astrocyte membrane resistance in parallel with Müller cell shunt resistance. The astrocyte component of the shunt at each node, AG, equals 1/25 of the membrane resistance of a single astrocyte. (An astrocyte is represented by 25 nodes.) The Müller cell component at each node represents a fraction of the shunt resistance of a single Müller cell (astrocyte-Müller cell junctional resistance in series with Müller cell membrane resistance). Best fits to the experimental data were obtained when 2.7 Müller cells were coupled to a single astrocyte. Thus, 0.108 (2.7/25) of a single Müller cell is coupled to each astrocyte node. Müller cells are not directly coupled to each other in the model as there is no evidence that such coupling exists in vivo (Robinson et al., 1993; Zahs and Newman, 1997).

When simulating whole-cell recording from a Müller cell, the shunt resistor, AMG, at a single astrocyte node is replaced by three resistors (Fig. 2A, red rectangles), representing the astrocyte-Müller cell shunt. Resistor AG represents astrocyte membrane resistance, resistor AM the astrocyte-Müller cell coupling resistance, and resistor MG the Müller cell membrane resistance. The node between resistors AM and MG (Fig. 2A, M) represents the intracellular compartment of the Müller cell.

Simulations of experiments are run by applying a 0.5-nA current to a single astrocyte node (A1) near the center of the array and measuring the resulting change in voltage in the eight adjacent astrocyte nodes or in the Müller cell node (M). For instance, when simulating coupling between astrocytes, current is injected at node $\mathrm{A} 1$ and the resulting voltage measured at nodes A2, A3, A4 ... A8. When simulating coupling between an astrocyte and a Müller cell, current is injected into an astrocyte node and the voltage measured at the Müller cell node, M. For simulations of Müller cell-toMüller cell coupling, shunt resistor AMG at a second astrocyte node is replaced with resistors $\mathrm{AG}, \mathrm{AM}$, and $M G$, with the values of $A M$ and $M G$ adjusted to represent a unitary Müller cell, rather than a fraction of a cell (Table 1). Current is injected into this Müller cell. Currents within the array approached zero well before they reached the edge of the array, demonstrating that boundary effects did not influence the simulation results.

Results of simulations were fit by eye to the experimental data. Values of AA, AMG, AG, AM, and MG were varied to obtain the best fit. Note that these 


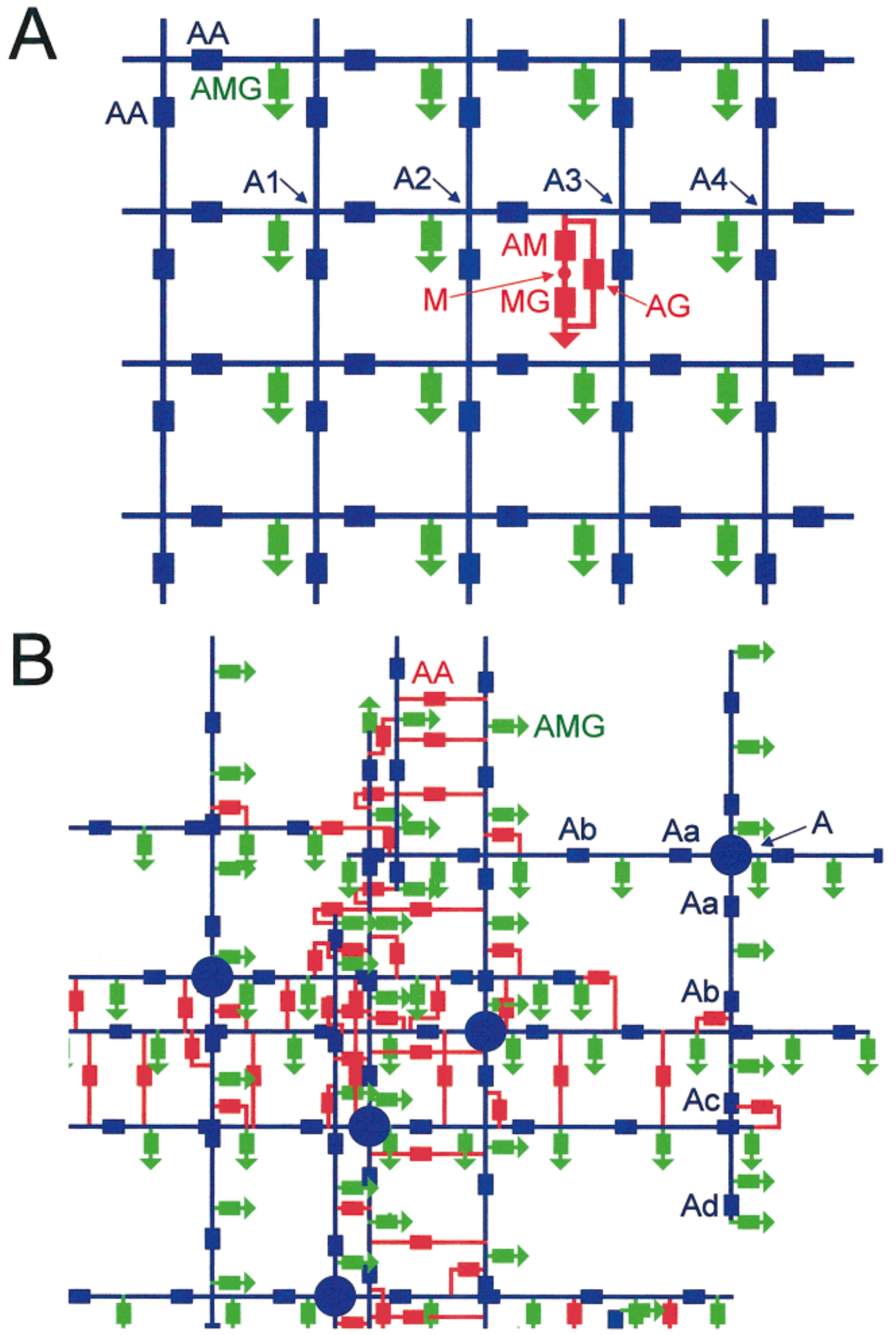

Fig. 2. Resistive models of glial cell coupling. A: Uniform resistive network model. A portion of the array is shown; the entire array consists of $24 \times 26$ nodes. Resistors AA (blue rectangles) represent internal astrocyte resistance and gap junctional coupling resistance; resistors AMG (green rectangles) represent astrocyte and Müller cell shunt resistances; resistor AG represents astrocyte membrane resistance; resistor AM represents astrocyte-Müller cell coupling resistance; resistor MG represents Müller cell membrane resistance. Nodes A1-A4 and M represent intracellular astrocyte and Müller cell compartments. B: Discrete cellular network model. Five of the 27 astrocytes in the model are shown. Resistors Aa, Ab, Ac, Ad (blue rectangles) represent internal cytoplasmic resistances of astrocyte processes; resistors AMG (green rectangles) represent astrocyte and Müller cell shunt resistances; resistors AA (red rectangles) represent gap junctional resistances coupling astrocytes together. Nodes A (blue circles) represent astrocyte soma compartments. Adjacent nodes in both models are spaced at $10-\mu \mathrm{m}$ intervals. Triangles represent the extracellular compartment (ground) in both diagrams. Values of the resistances are given in Table 1. 
TABLE 1. Values of parameters used in the resistive models of glial cell coupling*

\begin{tabular}{|c|c|c|c|c|}
\hline Parameter & Description & Control & $\begin{array}{l}1 \mathrm{mM} \\
\mathrm{Ba}^{2+}\end{array}$ & $\begin{array}{l}0.5 \mathrm{mM} \\
\text { octanol }\end{array}$ \\
\hline \multicolumn{5}{|c|}{$\begin{array}{l}\text { UNIFORM RESISTIVE NETWORK } \\
\text { (2.7 Müller cells per astrocyte; } 25 \text { nodes per a }\end{array}$} \\
\hline AA & $\begin{array}{l}\text { Combined astrocyte cytoplasmic resistance and astrocyte-astrocyte } \\
\text { coupling resistance }\end{array}$ & 100 & 400 & 5,000 \\
\hline $\mathrm{AMG}$ & $\begin{array}{l}\text { Combined shunt resistance per node (astrocyte membrane } \\
\text { resistance in parallel with Müller cell coupling and membrane } \\
\text { resistance); AMG = AG in parallel with (AM + MG) }\end{array}$ & 650 & 1,360 & 1,000 \\
\hline $\mathrm{AG}$ & Astrocyte membrane resistance per node & 5,000 & 10,000 & 5,000 \\
\hline $\mathrm{AM}$ & Astrocyte-Müller cell coupling resistance per node & 506 & 1081 & 908 \\
\hline \multirow{6}{*}{ MG } & Müller cell membrane resistance per node & 241 & 482 & 241 \\
\hline & Membrane resistance, single astrocyte & 200 & 400 & 200 \\
\hline & Astrocyte-Müller cell coupling resistance, single Müller cell & 55 & 117 & 99 \\
\hline & Membrane resistance, single Müller cell & 26 & 52 & 26 \\
\hline & Astrocyte input resistance & 41.8 & 145 & 586 \\
\hline & Müller cell input resistance & 20.6 & 44.4 & 25.4 \\
\hline
\end{tabular}

(2.0 Müller cells per astrocyte; 18 nodes per a (2.0 Müller cells per astrocyte; 18 nodes per astrocyte)

Astrocyte cytoplasmic resistances for 4 segments representing a cell 1 process (Aa is segment closest to soma)

$\mathrm{Ab}$

Ac

AA

AMG

Astrocyte-astrocyte coupling resistance per node

Combined shunt resistance per node (astrocyte membrane resistance in parallel with Müller cell coupling and membrane resistance); $\mathrm{AMG}=\mathrm{AG}$ in parallel with $(\mathrm{AM}+\mathrm{MG})$
Astrocyte membrane resistance per node

AG

Astrocyte-Müller cell coupling resistance per node

Müller cell membrane resistance per node

10

40
100

300

600

3,600

487

233

*All values are given in $\mathrm{M} \Omega$. The membrane resistance of single cells in the uniform resistive network model represents the total membrane resistance per cell (the resistance at all cell nodes in parallel). Input resistance of cells represents the resistance measured by current injection into a cell and incorporates coupling to the glial syncytium. Values of parameters AA, AG, AM, MG, as well as the number of Müller cells per astrocyte, were chosen to fit the experimental data. Cell membrane and input resistances and resistance AMG were determined from these values.

parameters are not independent, as AG in parallel with AM plus MG must equal AMG.

\section{Discrete cellular network model}

The uniform resistive network described above is a simplification of the actual glial network in the retina. The model does not distinguish between the internal resistance of astrocytes and the coupling resistance between astrocytes. Nor does it incorporate the variations in spacing between astrocytes that are seen in the retina (Fig. 1A). A discrete cellular network model (Fig. 2B) was developed to rectify these deficiencies.

The model consists of 27 discrete astrocytes. Each astrocyte is composed of a soma node (Fig. 2B, blue circles, A) connected to four radiating processes, each comprised of four resistors (Aa, Ab, Ac, and Ad) representing the internal resistances of four segments of the process. The four segments of each process represent compartments $10 \mu \mathrm{m}$ long and 3.0, 0.94, 0.48, and 0.30 $\mu \mathrm{m}$ in diameter (soma to tip), having an internal resistivity of $70 \Omega \cdot \mathrm{cm}$. Astrocytes near the center of the network are spaced close to each other $(10,15,20$, and $25 \mu \mathrm{m}$ apart), reflecting the close spacing of astrocytes often seen near blood vessels in the retina (Zahs and $\mathrm{Wu}, 2001$ ) (Fig. 1A). Astrocytes near the periphery of the model are spaced $50 \mu \mathrm{m}$ apart.

Each astrocyte node (the soma node as well as the nodes between each astrocyte resistor, $\mathrm{Aa}, \mathrm{Ab}, \mathrm{Ac}, \mathrm{Ad})$ is connected to ground by a shunt resistor (Fig. 2B, green rectangles, AMG) that represent astrocyte membrane resistance in parallel with Müller cell shunt resistance. As in the uniform resistive model, the shunt resistor $\mathrm{AMG}$ at a single astrocyte node is replaced by three resistors $\mathrm{AG}, \mathrm{AM}$, and $\mathrm{MG}$ (not shown in Fig. 2), when simulating coupling between an astrocyte and a Müller cell.

Coupling between astrocytes is simulated in the model by coupling resistors AA (Fig. 2B, red rectangles) that link processes of nearby astrocytes. A coupling resistor $\mathrm{AA}$ is inserted between nodes of neighboring astrocytes whenever the nodes are within $10 \mu \mathrm{m}$ of each other. Closely spaced astrocytes are coupled together by a greater number of resistors AA.

Simulations of coupling between astrocytes are run by applying a 0.5-nA current to the soma of one of the astrocytes and measuring the resulting change in voltage in all other astrocyte somata within an $80 \mu \mathrm{m}$ radius. Simulations of coupling between astrocytes and Müller cells are run by applying current to an astrocyte soma and measuring the voltage at the Müller cell node (the node between resistors AM and MG) for a Müller cell attached either to an astrocyte soma or to an astrocyte process.

\section{RESULTS}

The strength of electrical coupling between glial cells was assessed in whole cell current-clamp experiments. 
Recordings were made simultaneously from pairs of glial cells, either astrocyte-astrocyte, astrocyte-Müller cell, or Müller cell-Müller cell pairs. Recordings from a total of 205 cell pairs were used in this study.

Coupling between pairs of cells is sometimes determined by calculating the coupling ratio (Kettenmann and Ransom, 1988; Ransom and Kettenmann, 1990): the ratio of responses recorded from the injected and noninjected cells of a cell pair. In order to determine the response in the injected cell accurately, it is necessary to measure the voltage drop across the pipette used to inject the current. This proved difficult in our experiments, as the access resistance of the pipette was similar to the cell input resistance and often varied during the course of an experiment (Connors and Ransom, 1987).

Because coupling ratios could not be determined accurately, an alternate method was used to assess coupling between pairs of glial cells. A $0.5-\mathrm{nA}, 60-\mathrm{ms}$ current pulse was injected into one cell of a pair and the resulting voltage response recorded from the second, noninjected cell. The amplitude of this response was then plotted as a function of distance between the two cells. This procedure was repeated for many cell pairs separated by different distances. The distance between the tips of the two patch pipettes was taken as the intercellular distance.

This method of determining cell coupling assumes that the current-evoked voltage response in the injected cell is similar for all cell pairs tested. This is a good approximation, as we have previously shown that the input resistance of rat astrocytes and Müller cells varies little from cell to cell. Input resistance equaled $29 \pm 2$ and $24 \pm 2 \mathrm{M} \Omega$ in astrocytes and Müller cells, respectively (Zahs and Newman, 1997).

Representative voltage responses recorded from the noninjected cell of cell pairs are shown in Figure 3. The voltage deflection is largest for the astrocyte-astrocyte pair (Fig. 3A), smaller for the astrocyte-Müller cell pair (Fig. 3B), and smallest for the Müller cell-Müller cell pair (Fig. 3C). This pattern of response amplitudes was found in most recordings, demonstrating that electrical coupling between astrocytes is strongest, coupling between astrocytes and Müller cells somewhat weaker, and coupling between Müller cells weakest of all. [We assume that coupling between Müller cells is indirect and occurs by mutual coupling to the astrocyte network; there is no evidence that mammalian Müller cells are directly coupled (Robinson et al., 1993; Zahs and Newman, 1997).]

Controls were performed to ensure that the responses recorded from the noninjected cell were due to coupling between the cells. After recording from a cell pair, the current-passing pipette was withdrawn from the currentinjected cell, the electrode tip cleared of debris by applying positive pressure, and the pipette advanced to its original position. Current pulses were applied to the current-injection pipette and responses recorded from the noninjected cell of the pair. In all cases, the responses were either nondetectable or substantially smaller than

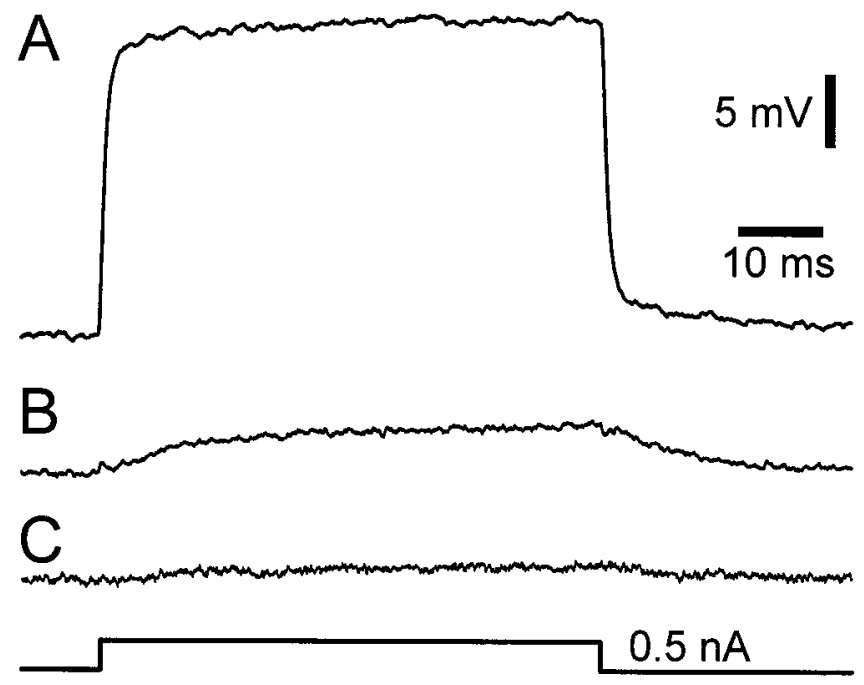

Fig. 3. Voltage responses of the noninjected cell evoked by current injection into the other cell of a cell pair. A: Astrocyte-astrocyte pair; 5.3- $\mu \mathrm{m}$ separation. B: Astrocyte-Müller cell pair. Current injected into astrocyte; voltage recorded from Müller cell; 8.0- $\mu \mathrm{m}$ separation. C: Müller cell-Müller cell pair; $5.9-\mu \mathrm{m}$ separation. Time course of 0.5-nA current pulse shown at the bottom.

the responses recorded when the current-passing pipette was attached to the current-injected cell. Responses of the noninjected cell averaged $0.09 \pm 0.02 \mathrm{mV}$ (85) in these control trials, compared with $1.63 \pm 0.29 \mathrm{mV}$ (95) for trials when the current-passing pipette was attached to the current-injected cell.

\section{Astrocyte-Astrocyte Coupling}

Coupling between pairs of astrocytes, plotted as a function of distance, is illustrated in Figure 4A-C. The data points indicate the amplitude of responses recorded from individual cells while the continuous lines show the fit provided by the uniform resistive network model (see below).

In control solution (Fig. 4A), the 0.5 -nA current pulse produced voltage responses of $\sim 10 \mathrm{mV}$ in the noninjected cell for astrocyte pairs spaced $\sim 10 \mu \mathrm{m}$ apart. As the distance between the cells increased, the amplitude of the responses decreased in a near-exponential fashion. There is a fair degree of scatter in the data points. The source of this scatter is discussed below.

Barium is an effective blocker of glial cell $\mathrm{K}^{+}$channels (Walz et al., 1984; Kettenmann and Ransom, 1988; Newman, 1989; Puro and Stuenkel, 1995) and increases the coupling ratio between glial cells in culture (Kettenmann and Ransom, 1988; Ransom and Kettenmann, 1990). The addition of $1 \mathrm{mM} \mathrm{Ba}{ }^{2+}$ to the superfusate resulted in larger voltage responses for cell pairs separated by $<40 \mu \mathrm{m}$ (Fig. 4B). These larger responses cannot be interpreted as an increase in coupling, however, as the increased membrane resistance produced by $\mathrm{Ba}^{2+}$ leads to larger voltage responses in the injected as well as the noninjected cell. Indeed, the fit 


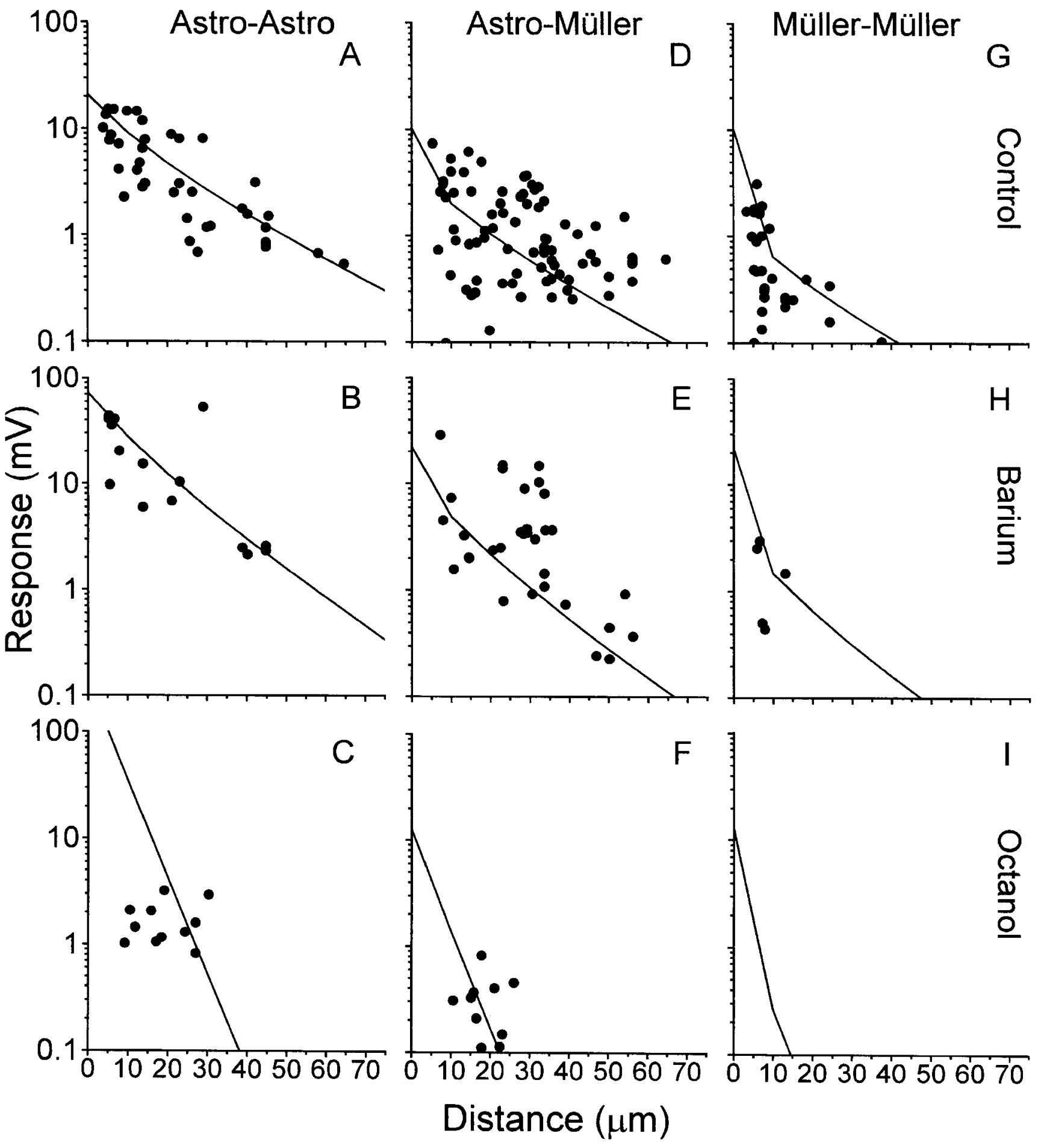

Fig. 4. Electrical coupling between glial cells. A-I: data points $(\bullet)$ show the amplitude of responses of the noninjected cell as a function of distance from the injected cell of cell pairs. Lines show the fit of the uniform resistive network model. The nine panels form an array of cell pair types (columns) and test conditions (top row, control; middle row, $1 \mathrm{mM} \mathrm{Ba}{ }^{2+}$; bottom row, $0.5 \mathrm{mM}$ octanol). Examples: A: Astrocyte-astrocyte coupling under control conditions; H: Müller cell-Müller cell coupling in the presence of barium. Data are plotted semilogarithmically; an exponential decline of response amplitude would appear as a straight line.

provided by the uniform resistive network model indicates that the falloff of response amplitude with distance was steeper after addition of $\mathrm{Ba}^{2+}$. The addition of $\mathrm{Ba}^{2+}$ depolarized astrocytes from a control level of
$-83.3 \pm 0.7 \mathrm{mV}(54)$ to $-58.7 \pm 2.1 \mathrm{mV}$ (54), indicating a block of cell $\mathrm{K}^{+}$channels.

Octanol reduces gap junctional coupling in many glial systems (Spray and Bennett, 1985; Giaume et al., 
1991a; Lee et al., 1994). In the retina, $0.5 \mathrm{mM}$ octanol blocks tracer coupling between astrocytes and Müller cells but is less effective in blocking coupling between astrocytes (Zahs and Newman, 1997). The addition of $0.5 \mathrm{mM}$ octanol reduced electrical coupling between astrocytes in the retina (Fig. $4 \mathrm{C}$ ). At a distance of $\sim 20$ $\mu \mathrm{m}$ between cells, response amplitudes were reduced $\sim 2.5$-fold by octanol.

\section{Astrocyte-Müller Cell Coupling}

Simultaneous recordings from astrocyte-Müller cell pairs were made to determine coupling between these two types of retinal glial cells. In some recordings, current was injected into the astrocyte and in others into the Müller cell. There was little difference in the resulting voltage responses recorded in the noninjected cell of the pair for the two sets of recordings. In addition, simulations using both the uniform resistive network and the discrete cellular network models demonstrated that current injection into either cell type should yield identical responses in the noninjected cell. Accordingly, experimental results from astrocyte and Müller cell injections were pooled.

In control solution, coupling between astrocytes and Müller cells (Fig. 4D) was somewhat weaker than was coupling between astrocytes. Mean response amplitude for pairs spaced 10-20 $\mu \mathrm{m}$ apart, for instance, was $1.8 \pm 0.4 \mathrm{mV}$ (19), compared with $8.6 \pm 1.4 \mathrm{mV}$ (9) in astrocyte pairs. In addition, there was a great deal more scatter in the data for astrocyte-Müller cell pairs.

The addition of $1 \mathrm{mM} \mathrm{Ba}{ }^{2+}$ (Fig. 4E) resulted in an increase in response amplitude, an effect similar to that seen in astrocyte-astrocyte pairs. For a given distance, the largest responses seen in the presence of $\mathrm{Ba}^{2+}$ were substantially greater than the largest responses seen in controls. As in the controls, there was considerable scatter in the data. Barium depolarized Müller cells from a control level of $-88.3 \pm 0.8 \mathrm{mV}$ (50) to $-43.1 \pm 2.6 \mathrm{mV}$ (50), indicating a substantial block of cell $\mathrm{K}^{+}$channels.

The addition of $0.5 \mathrm{mM}$ octanol reduced coupling substantially between astrocyte-Müller cell pairs (Fig. $4 \mathrm{~F})$. At $\sim 20 \mu \mathrm{m}$, responses were reduced $\sim 10$-fold by octanol, compared with controls.

\section{Müller Cell-Müller Cell Coupling}

Simultaneous recordings from pairs of Müller cells (Fig. 4G) show that coupling between Müller cells is substantially weaker than is coupling between astrocyte pairs or between astrocytes and Müller cells. Mean response amplitude for Müller cell pairs spaced 10-20 $\mu \mathrm{m}$ apart, for example, was $0.16 \pm 0.05 \mathrm{mV}$ (9), compared with $8.6 \mathrm{mV}$ for astrocyte pairs and $1.8 \mathrm{mV}$ for astrocyte-Müller cell pairs.

Little change in response amplitude was seen with the addition of $\mathrm{Ba}^{2+}$ (Fig. 4H), although the sample size was too small to compare the responses statistically. The addition of octanol eliminated coupling altogether (Fig. 4I). In the presence of $0.5 \mathrm{mM}$ octanol, no detectable response was recorded from the noninjected Müller cell.

\section{Rectification of Astrocyte-Müller Cell Coupling}

Tracer studies have demonstrated asymmetric chemical coupling between astrocytes and Müller cells in the rat retina (Zahs and Newman, 1997). Additional recordings were made from astrocyte-Müller cell pairs to determine whether the coupling between these cells showed electrical rectification. Data were collected from astrocyte-Müller cell pairs separated by $<27 \mu \mathrm{m}$. For each cell pair, both positive and negative $0.5-\mathrm{nA}$ current pulses were injected sequentially into both cells of the pair. For each pair, the Müller cell voltage response produced by positive current injection into the astrocyte was divided by the response produced by negative current injection. Similarly, the astrocyte responses produced by negative and positive current injection into the Müller cell were divided. These two ratios were averaged and represented a measure of rectification in the cell pair.

The mean response ratio for all astrocyte-Müller cell pairs examined was $1.10 \pm 0.11$ (14). This ratio is not statistically different from $1(P=0.35)$ indicating that coupling between astrocytes and Müller cells is not electrically rectifying.

\section{Uniform Resistive Network Simulations}

The uniform resistive network model (Fig. 2A), described under Resistive Models of Glial Cell Coupling (above), was used to simulate the results obtained in current injection experiments. The fits to the experimental data are shown by the lines in Figure 4. The values of the parameters used in the simulations are given in Table 1. A single set of values was used to fit astrocyte-astrocyte coupling, astrocyte-Müller cell coupling, and Müller cell-Müller cell coupling. The same changes in parameter values were used to model $\mathrm{Ba}^{2+}$ and octanol treatment for all three types of coupling.

The simulated responses obtained from the uniform resistive model would lie along a straight line when plotted semilogarithmically if coupling declined exponentially with distance. As shown previously (Jack et al., 1975; Lamb and Simon, 1976; Detwiler and Hodgkin, 1979; Mobbs et al., 1988), however, the decline of response amplitude with distance in a twodimensional resistive network is not described by a first-order exponential. The response initially declines more rapidly than an exponential, and has a slightly concave shape when plotted semilogarithmically. 
TABLE 2. Effective space constants of glial networks, determined by the uniform resistive network model ${ }^{*}$

\begin{tabular}{lccc}
\hline Condition & $\begin{array}{c}\text { Astrocyte- } \\
\text { astrocyte }\end{array}$ & $\begin{array}{c}\text { Astrocyte- } \\
\text { Müller cell }\end{array}$ & $\begin{array}{c}\text { Müller cell- } \\
\text { Müller cell }\end{array}$ \\
\hline Control & 12.9 & 6.2 & 3.7 \\
Barium $(1 \mathrm{mM})$ & 10.8 & 6.7 & 3.8 \\
Octanol $(0.5 \mathrm{mM})$ & 4.7 & 4.4 & 2.6 \\
\hline
\end{tabular}

*The effective space constant is defined as the distance from the point of current injection to the point where the voltage decreases to $1 / e$ of it maximal amplitude. All values are given in $\mu \mathrm{m}$.

\section{Control coupling}

Astrocyte-astrocyte coupling under control conditions is fit well by the uniform resistive model, with the model responses lying in the middle of the scatter of experimental values (Fig. 4A). The fit of the model for astrocyte-Müller cell (Fig. 4D) and Müller cell-Müller cell (Fig. 4G) coupling is not as good. The model values for astrocyte-Müller cell coupling are somewhat lower than the experimental values while the model values for Müller cell-Müller cell coupling are somewhat higher than the experimental values. This discrepancy could not be improved by varying model parameters.

Estimates of the decline of coupling with distance were based on model simulations. The glial cell network does not have a space constant, as commonly defined, as decay cannot be described by a first-order exponential. It is useful to calculate an "effective space constant," however, which we define as the distance from the point of current injection to the point where the voltage falls to $1 / e$. Note that because the relation deviates from an exponential, the voltage at a distance of two effective space constants will be greater than $1 / e^{2}$. [Technically, the space constant, $\lambda$, for a twodimensional resistive network is defined by the asymptote to the log response vs. distance plot at large distances (Jack et al., 1975). The effective space constant that we define is a more accurate measure of voltage decay for shorter, physiologically relevant distances.]

The effective space constant for astrocyte-astrocyte coupling, based on the fit provided by the uniform resistive model, was $12.9 \mu \mathrm{m}$ (Table 2). The effective space constants for coupling between astrocytes and Müller cells, and between Müller cells were even shorter, 6.2 and $3.7, \mu \mathrm{m}$ respectively.

The parameter values used to fit the experimental data indicate that 2.7 Müller cells, on average, are coupled to a single astrocyte. Each Müller cell has a membrane resistance of $26 \mathrm{M} \Omega$ and is coupled to an astrocyte by a gap junctional resistance of $55 \mathrm{M} \Omega$ (Table 1).

\section{Barium}

Experimental results obtained in the presence of barium (Fig. 4B,E,H) were best fit by the model when the following changes to model parameters were made: AA resistance (internal astrocyte resistance and astro- cyte-astrocyte coupling resistance) was raised from 100 to $400 \mathrm{M} \Omega$, AG resistance (astrocyte membrane resistance) was raised from 5,000 to $10,000 \mathrm{M} \Omega$, AM resistance (astrocyte to Müller cell coupling resistance) was raised from 506 to $1081 \mathrm{M} \Omega$, and $\mathrm{MG}$ resistance (Müller cell membrane resistance) was raised from 241 to $482 \mathrm{M} \Omega$ (Table 1). These changes produced adequate fits to the experimental data, although many of the data points for astrocyte-Müller cell coupling fell above the model values.

\section{Octanol}

Experimental results obtained with octanol (Fig. $4 \mathrm{C}, \mathrm{F}, \mathrm{I})$ were best fit by the model when the following changes were made: AA resistance was raised from 100 to 5,000 M $\Omega$; $\mathrm{AM}$ resistance was raised from 506 to 908 $\mathrm{M} \Omega$. AG and MG resistances were unchanged.

\section{Discrete Cellular Network Model Simulations}

Although the uniform resistive network model provides a good fit to the experimental data, the model does not account for the scatter seen in the results. This scatter could reflect an inherent variability of coupling in the glial cell network or it could be attributable to experimental measurement error or damage produced by the recording procedure.

A second model of the glial cell network, the discrete cellular network model, was developed to determine whether scatter is an intrinsic property of the network. The model, described in the Resistive Models section, above, is composed of 27 discrete astrocytes that are spaced at varying distances from each other (Fig. 2B). Cell processes are coupled to each other whenever individual cell nodes lie within $10 \mu \mathrm{m}$. The total coupling resistance between pairs of astrocytes ranged from 37.5 to $300 \mathrm{M} \Omega$, depending on the spacing between the cells. Astrocyte pairs separated by $>50 \mu \mathrm{m}$ were not directly coupled to each other. The best fit to the data was obtained when 2.0 Müller cells, on average, were coupled to a single astrocyte. As in the uniform resistive model, each Müller cell had a membrane resistance of $26 \mathrm{M} \Omega$ and was coupled to an astrocyte by a gap junctional resistance of $55 \mathrm{M} \Omega$.

The results of a simulation of astrocyte-astrocyte coupling under control conditions, along with the corresponding experimental data, are shown in Figure 5A. The results from the simulation (filled circles) show a similar degree of scatter as the experimental results (open triangles), with a wide range of responses seen.

A quantitative assessment of the scatter was made using linear regression analysis to fit log response vs. distance plots. The root-mean-squared (RMS) error for the best fits to the experimental and simulated results were nearly identical, 0.28 and 0.26 , respectively.

Results of a simulation of astrocyte-Müller cell coupling are shown in Figure 5B. In this case, the degree 


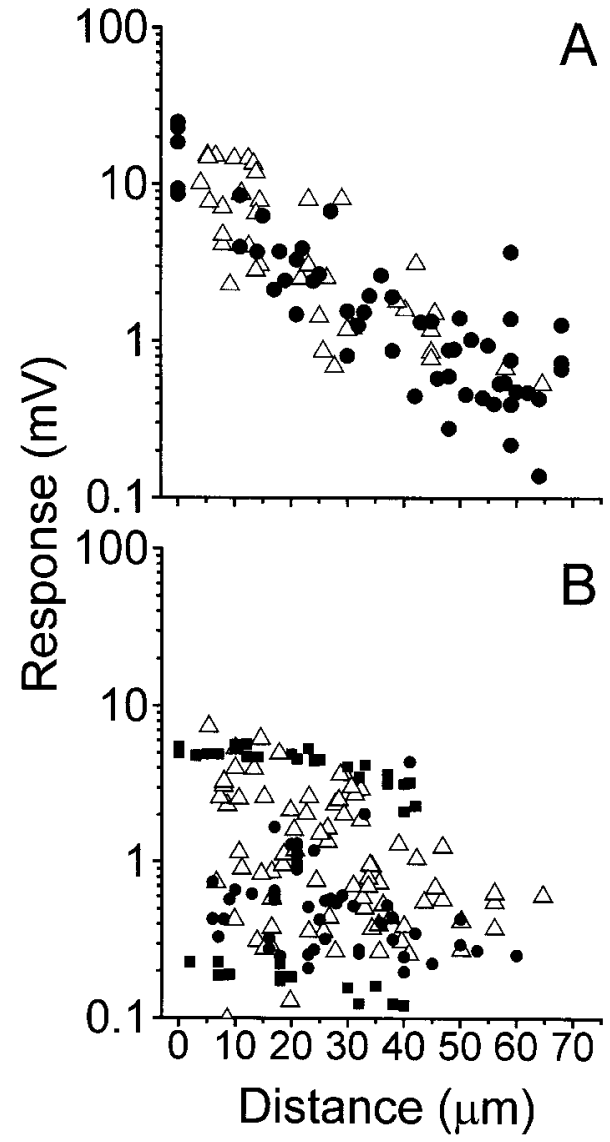

Fig. 5. Electrical coupling measured experimentally $(\triangle)$ and simulated by the discrete cellular network model $(\boldsymbol{\bullet}, \mathbf{\square})$. A: Astrocyteastrocyte coupling under control conditions. The variability in coupling in the experimental and simulation results are similar. Experimental data from Fig. 4A. B: Astrocyte-Müller cell coupling under control conditions. The variability in coupling in the simulation results is somewhat greater than it is in the experimental results. $\mathbf{\square}$, simulation results for Müller cells coupled directly to injected astrocytes; - simulation results for Müller cells indirectly coupled to injected astrocytes via other astrocytes. Experimental data from Fig. $4 \mathrm{D}$.

of scatter seen in the simulation results is somewhat greater than that in the experimental results. The RMS errors for linear regression fits to log response vs. distance plots were 0.35 and 0.53 for the experimental and simulated data, respectively.

The simulation provides an insight into why there is a large degree of scatter in the astrocyte-Müller cell results. Large responses, indicating close coupling, were seen in the simulation for some cell pairs when the Müller cell was directly coupled to an astrocyte (Fig. 5B, filled squares). Smaller responses, indicating weak coupling, were seen when a Müller cell was indirectly coupled to an astrocyte by connections through other astrocytes (Fig. 5B, filled circles).

\section{DISCUSSION}

We previously demonstrated by tracer injection that astrocytes are coupled to each other and to Müller cells in the rat retina (Zahs and Newman, 1997). The present study quantifies this coupling using electrical current flow as a measure of coupling strength.

\section{Space Constant}

Coupling strength can be characterized by the space constant of the glial cell network, a measure that reflects the distance that current spreads within the network. In the present study, an effective space constant was determined based on fits provided by the uniform resistive network model.

The effective space constant of the astrocyte network was $12.9 \mu \mathrm{m}$, much shorter than the average spacing of $\sim 50 \mu \mathrm{m}$ between astrocytes in the rat retina (ChanLing and Stone, 1991). The effective space constant of coupling between astrocytes and Müller cells was 6.2 $\mu \mathrm{m}$, while coupling from one Müller cell to another was $3.7 \mu \mathrm{m}$, shorter than the $\sim 9-\mu \mathrm{m}$ spacing between Müller cells (data from mouse) (Drager et al., 1984). These short space constants indicate that when a single retinal glial cell is depolarized in vivo (e.g., by a localized increase in extracellular $\mathrm{K}^{+}$), little current will be transferred to neighboring glial cells.

There are few published quantitative studies of coupling in glial networks in CNS tissue. A current-clamp study of coupling in amphibian Müller cells, which are directly coupled, demonstrated that these cells are weakly electrically coupled (Mobbs et al., 1988). The Müller cell network had a space constant of $15 \mu \mathrm{m}$. Coupling resistance between individual Müller cells was estimated to be $60 \mathrm{M} \Omega$. A second current injection study, in ependymal glial cells of the turtle cortex, also found weak coupling (Connors and Ransom, 1987). Current spread was seen for closely spaced cell pairs, but coupling could not be detected for cells spaced more than $50 \mu \mathrm{m}$ apart, indicating a space constant substantially shorter than $50 \mu \mathrm{m}$.

Space constants of networks presumed to be coupled glial cells have also been estimated indirectly, by measuring changes in extracellular $\mathrm{K}^{+}$evoked by currents applied across the cortical surface. Space constants of 200 and $60 \mu \mathrm{m}$ were calculated for rat (Gardner-Medwin and Nicholson, 1983) and frog (Gardner-Medwin, 1985) cortex, respectively. It is not clear whether these larger space constant values reflect more extensive coupling of glial cells in the cortex or are inaccurate measures resulting from the limitations in the methods used to estimate coupling.

\section{Astrocyte-Müller Cell Rectification}

Our tracer study of coupling in the rat retina (Zahs and Newman, 1997) demonstrated asymmetric coupling between astrocytes and Müller cells. Neurobiotin passed from astrocytes to Müller cells but not noticeably from Müller cells to astrocytes. Similar hetero- 
typic coupling between astrocytes and Müller cells was seen in the rabbit retina (Robinson et al., 1993).

The present study demonstrates that this heterotypic junction is not electrically rectifying. Currents passed equally well in both directions. This is not altogether surprising. Large tracer molecules that are near the size limit for passage through gap junctions may encounter asymmetric free-energy barriers in heterotypic junctions, whereas small ions, which carry current, will pass through these junctions unhindered (Flagg-Newton and Loewenstein, 1980; Loewenstein, 1981). The asymmetric coupling (chemical rectification) seen at these junctions could be due to this freeenergy barrier asymmetry and to the resulting asymmetric saturation of passive diffusion through the channels (Loewenstein, 1981). [Asymmetric coupling cannot be due to hypothetical unidirectional channels, however, which violate the Second Law of Thermodynamics (Meister, 1994; Buehler, 1994).] Alternatively, asymmetric coupling could result from the asymmetric geometry of the astrocyte-Müller cell syncytium.

\section{Variability of Coupling}

Substantial variability is present in the experimental responses, with the scatter seen in astrocyte-Müller cell responses substantially larger than that seen in astrocyte-astrocyte responses. Similar variability is seen in electrical coupling between amphibian Müller cells (Mobbs et al., 1988) and in coupled glial cells in culture (Kettenmann and Ransom, 1988; Ransom and Kettenmann, 1990).

Simulations using the discrete cellular network model demonstrate that the response variability seen in our preparation is an intrinsic property of the retinal glial cell network. Indeed, the somewhat simplistic representation of glial cell connections provided by the discrete cellular network model closely reproduces the variability in coupling seen in the experimental results. For astrocyte-astrocyte coupling, the RMS error of the experimental and simulation responses were nearly identical. For astrocyte-Müller cell coupling, the scatter seen in the simulation results was actually larger than that of the experimental results. A discrete cellular network model that more accurately reflects the geometry of retinal glial cells (one having more astrocyte processes contacting one another) would, most likely, reproduce the variability in coupling more accurately.

The scatter seen in astrocyte-astrocyte coupling arises because some astrocyte pairs are coupled to each other by more gap junctions than are other pairs. The larger degree of scatter seen in astrocyte-Müller cell pairs arises for a different reason. Strong coupling is present when a Müller cell is attached directly to an astrocyte. Much weaker coupling is seen when a Müller cell and an astrocyte are coupled indirectly through other astrocytes.

\section{Predictions of Model}

The results of the simulations provide a number of predictions. Some of these predictions can be compared with published results, whereas others can be tested in future experiments.

\section{Glial cell membrane resistance and numbers}

The values of resistors AA, AMG, AG, AM, and MG chosen to fit the experimental data in the uniform resistive model yield input resistance values of 41.8 and 20.6 M , respectively, for astrocytes and Müller cells. By comparison, the input resistance of astrocytes and Müller cells measured experimentally in the rat retina is 29 and $24 \mathrm{M} \Omega$, respectively (Zahs and Newman, 1997). The input resistance of freshly dissociated mouse Müller cells is $14.6 \mathrm{M} \Omega$ (Newman, 1987).

Best fits to the experimental data were obtained when a total of 2.7 (uniform resistive model) or 2.0 (discrete cellular model) Müller cells were coupled to each astrocyte. These ratios were derived independently for the two models and their similarity suggests that they represent an accurate estimate of Müller cell-astrocyte coupling in the retina. By comparison, a recent tracer study (Zahs and $\mathrm{Wu}, 2001$ ) indicates that 3-6 Müller cells are coupled to each astrocyte in the rat retina (depending on how close the cells lie to blood vessels).

\section{Gap junctional coupling resistance}

Closely spaced astrocytes in the discrete cellular network model were coupled by junctional resistances as low as $37.5 \mathrm{M} \Omega$. This value is similar to the lowest junctional resistances observed in pairs of astrocytes in culture: $31 \mathrm{M} \Omega$ (Dermietzel et al., 1991) and $50 \mathrm{M} \Omega$ (Giaume et al., 1991b).

Experimental results were fit in both models using a coupling resistance of $55 \mathrm{M} \Omega$ between Müller cells and the astrocyte network. This value is similar to the calculated coupling resistance between Müller cells in the salamander retina: $60 \mathrm{M} \Omega$ (Mobbs et al., 1988).

\section{Barium}

The effect of $1 \mathrm{mM} \mathrm{Ba}^{2+}$ on glial cell coupling was simulated in the uniform resistive model by increasing resistance AA 4-fold, increasing resistance AG (astrocyte membrane resistance) 2-fold, increasing resistance AM (astrocyte-Müller cell coupling resistance) 2.1-fold, and increasing MG (Müller cell membrane resistance) 2 -fold. $\mathrm{As} \mathrm{Ba}^{2+}$ is not likely to affect astrocyte cytoplasmic resistance, the 4-fold increase in AA likely reflects an increase in astrocyte-astrocyte coupling resistance. The experimental data were fit less well if $\mathrm{Ba}^{2+}$ was assumed to increase astrocyte and 
Müller cell membrane resistances to a greater extent and to leave gap junctional resistances unchanged. Thus, the simulation predicts that gap junctional resistances between astrocytes and between astrocytes and Müller cells are increased by $\mathrm{Ba}^{2+}$.

These predicted $\mathrm{Ba}^{2+}$ effects are somewhat unexpected. $\mathrm{Ba}^{2+}$ is known to block glial cell $\mathrm{K}^{+}$channels (Newman, 1989; Reichelt and Pannicke, 1993; Puro and Stuenkel, 1995) but should not block gap junctions. Barium may not be blocking gap junctions directly, however. Application of $1 \mathrm{mM} \mathrm{Ba}{ }^{2+}$ will depolarize both astrocytes and Müller cells dramatically (Kettenmann and Ransom, 1988; Newman, 1989; Reichelt and Pannicke, 1993) and could lead to substantial increases in intracellular $\mathrm{Ca}^{2+}$ by opening voltage-gated $\mathrm{Ca}^{2+}$ channels, which are present in both astrocytes (MacVicar, 1984; Barres et al., 1990) and Müller cells (Newman, 1985; Puro et al., 1996). The $\mathrm{Ba}^{2+}$-induced depolarization or the increased $\mathrm{Ca}^{2+}$ could result in a block of gap junction channels.

Barium should increase astrocyte and Müller cell membrane resistance, but to a greater extent than the 2 -fold increase indicated by the model. In different species, $1 \mathrm{mM} \mathrm{Ba}^{2+}$ raises cell input resistance to varying degrees. In dissociated human Müller cells, resistance is raised $\sim 5$-fold (Francke et al., 1997; Puro and Stuenkel, 1995), in guinea pig, between $\sim 7.5$-fold (Reichelt and Pannicke, 1993) and 89-fold (Francke et al., 1995), and in salamander $\sim 20$-fold (Newman, 1989).

\section{Octanol}

The effect of $0.5 \mathrm{mM}$ octanol on glial cell coupling was simulated in the uniform resistive model by increasing resistance AA 50-fold and increasing resistance AM 1.8-fold. Resistances AG and MG were unchanged in the simulation.

These increases in coupling resistances are consistent with the known effect of octanol to block gap junctions (Spray and Bennett, 1985; Giaume et al., 1991a; Lee et al., 1994). It is unlikely, however, that octanol blocks astrocyte-astrocyte junctions to a far greater extent than astrocyte-Müller cell junctions, as indicated by the simulation. Indeed, our tracer study of coupling in the rat retina (Zahs and Newman, 1997) suggests that octanol is more effective in blocking astrocyte-Müller cell junctions than it is in blocking astrocyte-astrocyte junctions.

\section{Summary}

The discrepancies between previously published experimental data and the predictions of the resistive models demonstrate that the models do not represent a complete description of electrical coupling between retinal glial cells. The uniform resistive model is clearly an oversimplification of the glial network. Although it represents the glial network in greater detail, the discrete cellular network model also oversimplifies the morphological relations between astrocytes.

Despite these shortcomings, the models do accurately account for many of the properties of electrical coupling in the glial cell network and offer useful insights. Coupling between astrocytes, between astrocytes and Müller cells, and between Müller cells (under control conditions and in the presence of $\mathrm{Ba}^{2+}$ and octanol) are all fit by the uniform resistive network model using a single set of parameter values. These fits provide an estimate of the effective space constants for coupling in the glial network. In addition, the variation in coupling strength seen in the experimental results is accurately simulated by the discrete cellular network model, demonstrating that this variability is an intrinsic property of the network. Model simulations also suggest that in addition to raising cell membrane resistance, $\mathrm{Ba}^{2+}$ may also raise coupling resistance in glial cell networks.

\section{Functions of Glial Coupling in the Retina}

Glial cell coupling is believed to enhance the regulation of $\mathrm{K}^{+}$in the CNS by allowing spatial buffer currents to pass between cells in glial cell networks (Orkand et al., 1966; Newman, 1995). Given the short space constants found in this study, the contribution of coupling to the spread of spatial buffer currents in the retina will be minimal. The effective space constant of coupling between astrocytes, $13 \mu \mathrm{m}$, is much shorter than the spacing between astrocytes and little spatial buffer current will spread to adjacent cells. Similarly, the lateral spread of spatial buffer current between Müller cells will be minimal, given the short, $4 \mu \mathrm{m}$, space constant between these cells. Similar conclusions were reached in analyses of spatial buffering in the amphibian retina (Gardner-Medwin, 1986; Mobbs et al., 1988). As suggested previously (Newman et al., 1984; Karwoski et al., 1989), the primary spatial buffer pathway within the retina is along the length of single Müller cells and does not involve gap junctional connections to other glial cells.

The absence of significant spread of spatial buffer current between retinal glial cells, despite the high degree of coupling between these cells, can, most likely, be attributed to the shunting effect of the low resistance membranes of glial cells. Both astrocytes and Müller cells have high $\mathrm{K}^{+}$membrane conductances, and most spatial buffer current will flow out through these conductances rather than spreading into neighboring glial cells through gap junctions.

In contrast to electrical coupling, chemical coupling between astrocytes is sufficiently strong to mediate propagation of intercellular signals. A recent study of $\mathrm{Ca}^{2+}$ wave propagation in retinal glial cells of the rat (Newman, 2001a) demonstrates that propagation can occur by the spread of an intracellular messenger, presumably $\mathrm{IP}_{3}$, through gap junctions linking astrocytes. 
Chemical coupling between glial cells is effective because internal messengers, such as $\mathrm{IP}_{3}$, can diffuse through gap junctions but cannot flow out of the cells across the plasma membrane. Internal messenger concentration thus remains high as it diffuses via gap junctions through the glial cell network.

Gap junctions may also serve as a pathway for the spread of metabolites and ions between glial cells (Loewenstein, 1981). In cultured rat hippocampal astrocytes, gap junctional coupling functions to equalize intracellular $\mathrm{Na}^{+}$levels between cells (Rose and Ransom, 1997). A similar spread of ions and small molecules may occur between glial cells in the mammalian retina. This coupling could serve to enhance the transport of key metabolites, such as glutamate, glutamine, and lactate, both into and out of glial cells, by allowing them to diffuse between neighboring cells in the glial syncytium.

\section{ACKNOWLEDGMENTS}

The authors thank Kathleen R. Zahs for the generous use of the images in Figure 1 and Janice I. Gepner for helpful comments on the manuscript.

\section{REFERENCES}

Barres BA, Koroshetz WJ, Chun LLY, Corey DP. 1990. Ion channel expression by white matter glia: the type-1 astrocyte. Neuron 5:527-544.

Buehler L. 1994. Gap junctions and intercellular communications. Science 265:1018-1019.

Chan-Ling T, Stone J. 1991. Factors determining the morphology and distribution of astrocytes in the cat retina: a "contact-spacing" model of astrocyte interaction. J Comp Neurol 303:387-399.

Connors BW, Ransom BR. 1987. Electrophysiological properties of ependymal cells (radial glia) in dorsal cortex of the turtle, Pseudemys scripta. J Physiol 385:287-306.

Dermietzel R, Hertzberg EL, Kessler JM, Spray DC. 1991. Gap junctions between cultured astrocytes: immunocytochemical, molecular, and electrophysiological analysis. J Neurosci 11:1421-1432.

Detwiler PB, Hodgkin AL. 1979. Electrical coupling between cones in turtle retina. J Physiol 291:75-100.

Drager UC, Edwards DL, Barnstable CJ. 1984. Antibodies against filamentous components in discrete cell types of the mouse retina. J Neurosci 4:2025-2042.

Enkvist MOK, McCarthy KD. 1992. Activation of protein kinase C blocks astroglial gap junction communication and inhibits the spread of calcium waves. J Neurochem 59:519-526.

Finkbeiner SM. 1993. Glial calcium. Glia 9:83-104.

Flagg-Newton JL, Loewenstein WR. 1980. Asymmetrically permeable membrane channels in cell junction. Science 207:771-773.

Francke M, Pannicke T, Reichelt W. 1995. Repetitive depletion and recovery of intracellular $\mathrm{K}^{+}$in retinal Müller glial cells during whole-cell voltage-clamp. J Neurosci Methods 61:169-184.

Francke M, Pannicke T, Biedermann B, Faude F, Wiedemann P, Reichenbach A, Reichelt W. 1997. Loss of inwardly rectifying potassium currents by human retinal glial cells in diseases of the eye. Glia 20:210-218.

Gardner-Medwin AR. 1985. The space constant for glial potassium buffering in the isolated frog brain. J Physiol 365:52P.

Gardner-Medwin AR. 1986. A new framework for assessment of potassium-buffering mechanisms. Ann NY Acad Sci 481:287-302.

Gardner-Medwin AR, Nicholson C. 1983. Changes of extracellular potassium activity induced by electric current through brain tissue in the rat. J Physiol 335:375-392.

Giaume C, Marin P, Cordier J, Glowinski J, Premont J. 1991a. Adrenergic regulation of intercellular communications between cul- tured striatal astrocytes from the mouse. Proc Natl Acad Sci USA 88:5577-5581.

Giaume C, Fromaget C, El Aoumari A, Cordier J, Glowinski J, Gros G. 1991b. Gap junctions in cultured astrocytes: single channel currents and characterization of channel-forming protein. Neuron 6:133-143.

Jack JJB, Noble D, Tsien RW. 1975. Electric current flow in excitable cells. Oxford: Clarendon Press.

Karwoski CJ, Lu H-K, Newman EA. 1989. Spatial buffering of lightevoked potassium increases by retinal Müller (glial) cells. Science 244:578-580.

Kettenmann H, Ransom BR. 1988. Electrical coupling between astrocytes and between oligodendrocytes studied in mammalian cell cultures. Glia 1:64-73.

Lamb TD, Simon EJ. 1976. The relation between intercellular coupling and electrical noise in turtle photoreceptors. J Physiol 263: 257-286.

Lee SH, Kim WT, Cornell-Bell AH, Sontheimer H. 1994. Astrocytes exhibit regional specificity in gap-junction coupling. Glia 11:315325.

Loewenstein WR. 1981. Junctional intercellular communication: the cell-to-cell membrane channel. Physiol Rev 61:829-913.

MacVicar BA. 1984. Voltage-dependent calcium channels in glial cells. Science 226:1345-1347.

Meister M. 1994. Gap junctions and intercellular communications. Science 265:1018.

Mobbs P, Brew H, Attwell D. 1988. A quantitative analysis of glial cell coupling in the retina of the axolotl (Ambystoma mexicanum). Brain Res 460:235-245.

Newman EA. 1985. Voltage-dependent calcium and potassium channels in retinal glial cells. Nature 317:809-811.

Newman EA. 1987. Distribution of potassium conductance in mammalian Müller (glial) cells: a comparative study. J Neurosci 7:24232432.

Newman EA. 1989. Potassium conductance block by barium in amphibian Müller cells. Brain Res 498:308-314.

Newman EA. 1995. Glial cell regulation of extracellular potassium. In: Kettenmann H, Ransom BR, editors. Neuroglia. New York: Oxford University Press; p 717-731.

Newman EA. 2001a. Propagation of intercellular calcium waves in retinal astrocytes and Müller cells. J Neurosci 21:2215-2223.

Newman EA. 2001b. Glia of the retina. In: Ryan SJ, editor. Retina. St. Louis, MO: CV Mosby; p 89-103.

Newman EA, Frambach DA, Odette LL. 1984. Control of extracellular potassium levels by retinal glial cell $\mathrm{K}^{+}$siphoning. Science 225 : $1174-1175$

Orkand RK, Nicholls JG, Kuffler SW. 1966. Effect of nerve impulses on the membrane potential of glial cells in the central nervous system of amphibia. J Neurophysiol 29:788-806.

Puro DG, Stuenkel EL. 1995. Thrombin-induced inhibition of potassium currents in human retinal glial (Müller) cells. J Physiol 485.2: 337-348.

Puro DG, Hwang J-J, Kwon O, Chin H. 1996. Characterization of an L-type calcium channel expressed by human retinal Müller (glial) cells. Mol Brain Res 37:41-48.

Ransom BR. 1995. Gap junctions. In: Kettenmann H, Ransom B, editors. Neuroglia. New York: Oxford University Press. p 299-318.

Ransom BR, Kettenmann H. 1990. Electrical coupling, without dye coupling, between mammalian astrocytes and oligodendrocytes in cell culture. Glia 3:258-266.

Reichelt W, Pannicke T. 1993. Voltage-dependent $\mathrm{K}^{+}$currents in guinea pig Müller (glial) cells show different sensitivities to blockade by $\mathrm{Ba}^{2+}$. Neurosci Lett 155:15-18.

Robinson SR, Hampson ECGM, Munro MN, Vaney DI. 1993. Unidirectional coupling of gap junctions between neuroglia. Science 262 : 1072-1074.

Rose CR, Ransom BR. 1997. Gap junctions equalize intracellular $\mathrm{Na}^{+}$ concentration in astrocytes. Glia 20:299-307.

Spray DC, Bennett MVL. 1985. Physiology and pharmacology of gap junctions. Annu Rev Physiol 47:281-303.

Von Blankenfeld G, Ransom BR, Kettenmann H. 1993. Development of cell-cell coupling among cells of the oligodendrocyte lineage. Glia 7:322-328

Walz W, Shargool M, Hertz L. 1984. Barium-induced inhibition of $\mathrm{K}^{+}$ transport mechanisms in cortical astrocytes-its possible contribution to the large $\mathrm{Ba}^{2+}$-evoked extracellular $\mathrm{K}^{+}$signal in brain. Neurosci 13:945-949.

Zahs KR, Newman EA. 1997. Asymmetric gap junctional coupling between glial cells in the rat retina. Glia 20:10-22.

Zahs KR, Wu T. 2001. Confocal microscopic study of glial-vascular relationships in the retinas of pigmented rats. J Comp Neurol 429:253-269. 